\title{
First total synthesis of yanuthones: novel farnesylated epoxycyclohexenoid marine natural products
}

\author{
Goverdhan Mehta* and Subhas Chandra Pan \\ Department of Organic Chemistry, Indian Institute of Science, Bangalore 560 012, India
}

\begin{abstract}
The total synthesis of the recently isolated marine natural products of mixed biosynthetic origin, yanuthones A, B, C and 22-deacylyanuthone A, has been accomplished following a short regio- and stereocontrolled approach involving the key intermediacy of 2-farnesyl- $p$-benzoquinone.
\end{abstract}

Hybrid natural products derived through mixed biosynthetic pathways evoke intrinsic synthetic interest as they embody diverse structural fragments. ${ }^{1}$ In the year 2000 , Ireland and co-workers ${ }^{2}$ reported the isolation and structure elucidation of eight bioactive farnesylated epoxy cyclohexenoids $\mathbf{1}-\mathbf{8}$ with various oxy-substitution patterns from an Aspergillus niger isolate obtained from tissue homogenates of an orange Aplidium sp. ascidian. These natural products were named yanuthones $\mathrm{A}-\mathrm{E}$, and derivatives, as shown in Chart 1. They exhibited weak antimicrobial activity against methicillin resistant Staphylococcus aureus and vancomycin resistant Enterococcus. More recently, deacetoxyyanuthone A 9, a close congener of 1-8, along with other related compounds has been isolated from a marine isolate of genus Penicillium sp. and shown to display mild antibacterial activity against methicillin resistant and multidrug resistant S. aureus (MIC, $50 \mu \mathrm{g} / \mathrm{ml}){ }^{3}$

There have also been earlier literature reports of the isolation of farnesylated epoxycyclohexenoid natural products derived through the combination of the shikimate and mevalonate pathways. ${ }^{2-5}$ For example, the research groups of Anke and co-workers ${ }^{4}$ and Rickards and co-workers ${ }^{5}$ have reported the structure elucidation of the oligosporon group of antibiotics, for example, oligosporon 10 and oligosporol B 11 (Chart 1) from the culture strains of Arthrobotrys oligospora from Netherlands and Australian isolates, respectively. It

\footnotetext{
*Corresponding author. Tel.: +91 8022932850; fax: +91 8023600936; e-mail: gm@orgchem.iisc.ernet.in
}

was further shown that the oligosporon group of natural products exhibit antibacterial, cytotoxic and nematocidal effects. The structural uniqueness of these farnesylated epoxyquinone natural products coupled with their diverse occurrence and wide ranging bioactivity profile aroused our synthetic interest. In this letter, we disclose a simple and general strategy that has resulted in the first total synthesis of several yanuthones in racemic form.

In formulating a general synthetic approach to yanuthones and other farnesylated epoxycyclohexenoid natural products, Chart 1 , we recognized the pivotal role of 2-farnesyl-p-benzoquinone $\mathbf{1 2}$ as the starting point. Quite surprisingly, while $\mathbf{1 2}$ has been found in Nature, ${ }^{6}$ its synthesis, to our knowledge, has only been reported once. $^{7 \mathrm{a}}$ There is also a report in the patent literature ${ }^{7 \mathrm{~b}}$ following a somewhat circuitous route. Our initial engagement therefore was to devise a convenient access to $\mathbf{1 2}$ from readily available starting materials and this objective was achieved following Yamamoto's recent adaptation $^{8 \mathrm{a}}$ of a previously described protocol $^{8 \mathrm{~b}}$ for the direct dehydrative coupling between polyprenyl alcohols and phenols. Thus, when a mixture of commercially available $p$-methoxyphenol 13 and $(E, E)$-farnesol 14 was exposed to scandium(III) triflate $(30 \mathrm{~mol} \%)$, 2-farnesylhydroquinone monomethyl ether $\mathbf{1 5}$ was obtained (Scheme 1). Ceric ammonium nitrate (CAN) oxidation of $\mathbf{1 5}$ delivered the key 2-farnesyl-p-benzoquinone $\mathbf{1 2}^{9}$ precursor smoothly and in just two steps (Scheme 1).

The next task was to elaborate the quinone moiety present in $\mathbf{1 2}$ to the requisite epoxy-cyclohexenoid functional array present in yanuthones $\mathbf{1 - 8}$, in a stereocontrolled 
<smiles>[R2]CC1=CC(=O)[C@@]2(C/C=C(\C)CC/C=C(\C)CC/C=C(\C)C[R])O[C@@H]2C1[R]</smiles>

$\begin{array}{cccc}\text { Compound } & \mathrm{R}^{1} & \mathrm{R}^{2} & \mathrm{R}^{3} \\ \text { Yanuthone A 1 } & \mathrm{H} & -\mathrm{OH},--\mathrm{H} & \mathrm{Ac} \\ \text { Yanuthone B 2 } & \mathrm{H} & \mathrm{O} & \mathrm{Ac} \\ \text { Yanuthone C 3 } & \mathrm{H} & -\mathrm{OAc},--\mathrm{H} & \mathrm{OH},--\mathrm{H} \\ \text { Yanuthone D 4 } & \mathrm{H} & \mathrm{O} \\ \text { 1-Hydroxy A 6 } & \mathrm{OH} & -\mathrm{OH},--\mathrm{H} \\ \text { 1-Hydroxy C 7 } & \mathrm{OH} & -\mathrm{OAc},--\mathrm{H}\end{array}$<smiles>CC(C)=CCC/C(C)=C/CC/C(C)=C/C[C@@]12O[C@@H]1[C@H](O)C(C)=CC2=O</smiles><smiles>CC(=O)OCC1=C[C@@H](O)[C@]2(C(O)C=C(C)C=CC=C(C)CCC=C(C)C)O[C@@H]2C1=O</smiles><smiles>CC(=O)OCC1=C[C@@H](O)[C@]2(C(O)C=C(C)C=CC=C(C)CCC=C(C)C)O[C@@H]2[C@H]1O</smiles>

Chart 1.

manner. For this purpose, a tactic recently highlighted by us ${ }^{10}$ in the syntheses of several epoxyquinone natural products was adopted. Thus, Diels-Alder reaction of 2farnesyl-p-quinone $\mathbf{1 5}$ with cyclopentadiene was regioand stereoselective and furnished the endo-adduct $16^{9}$ in good yield (Scheme 1). Base-mediated epoxidation on the enedione moiety in topologically biased 16 occurred as anticipated from the convex face to furnish exclusively the exo-epoxyquinone derivative 17. DBUmediated hydroxymethylation of $\mathbf{1 7}$ was stereoselective (exo-face preference) and regioselective (10:1) with predominant $\mathrm{C}-\mathrm{C}$ bond formation 'para' with respect to the farnesyl chain to furnish hydroxymethyl dione 18. ${ }^{9}$ At this stage, a quick check was made on the efficacy of our strategy by subjecting the tricyclic dione $\mathbf{1 8}$ to retro-Diels-Alder reaction to furnish enedione 19,9 acetylation of which led to $\mathbf{2}$ whose spectral data were found to be identical ${ }^{2,9}$ with those reported for the natural product yanuthone B (Scheme 1).

Encouraged by this convenient access to the natural product yanuthone B, we ventured to extend our strategy to other yanuthones, which besides other functional group adjustments required installation of the requisite hydroxyl group stereochemistry at C-15 corresponding to the natural products. Taking a cue from our recent contra-intuitive observation, ${ }^{11}$ the tricyclic hydroxydione 18 was subjected to DIBAL-H reduction, employing two equivalents of the reagent, to furnish the exo-hydroxy product $\mathbf{2 0}$ in a remarkable regio- and stereocontrolled operation $^{9,12}$ (Scheme 2). Retro-DielsAlder reaction in $\mathbf{2 0}$ led to $\mathbf{8}$, which was found to be spectroscopically identical ${ }^{2,9}$ with the natural product 22-deacetylyanuthone A. Further, when $\mathbf{8}$ was subjected to an acyl transfer reaction under a carefully controlled regime (low temperature and close TLC monitoring), a readily separable mixture (2:1) of yanuthone $A \mathbf{1}$ and yanuthone C $\mathbf{3}$ was formed. The spectral data of our synthetic $\mathbf{1}$ and $\mathbf{3}$ were found to be identical with those reported for the natural products (Scheme 2).

The relative stereochemistry of the yanuthones and other compounds displayed in Chart 1, particularly the C-15 hydroxyl stereochemistry, has been primarily assigned $^{2-4}$ on the basis of the $J_{\mathrm{H}-14, \mathrm{H}-15}$ coupling constants, which typically were found to be in the range of $2.5-3.0 \mathrm{~Hz}$ for the cis-stereochemical disposition. While comparison of these $J$ values was made ${ }^{2}$ with some model epoxycyclohexenones having a cis-disposition, the coupling constant data for the corresponding compounds with $\mathrm{H}-14$ and $\mathrm{H}-15$ in a trans-relationship were not available. We therefore decided to further secure the stereochemical assignment of the C-15 hydroxyl in the yanuthones by preparing the $\mathrm{C}-15$ epimers of yanuthones $\mathbf{1}, \mathbf{3}$ and $\mathbf{8}$ for comparison purposes. Towards this end, enedione 19 was reduced with DIBAL-H to furnish 22-deacetyl-epi-yanuthone A 21 in a regio- and stereocontrolled reduction (Scheme 3). ${ }^{13}$ The opposite stereochemical outcome during the DIBAL-H reduction of tricyclic 18 and monocyclic 19 underscores the importance of tight stereoelectronic control, engendered by the vicinal environment for aluminium coordination, in these reductions. Carefully controlled acetylation of $\mathbf{2 1}$ furnished a mixture of epiyanuthone A 22 and epi-yanuthone C 23 (2:1). The $J_{\mathrm{H}-14, \mathrm{H}-15}$ coupling constants in the epi-series 21-23 were 
<smiles>COc1ccc(O)cc1</smiles>

13
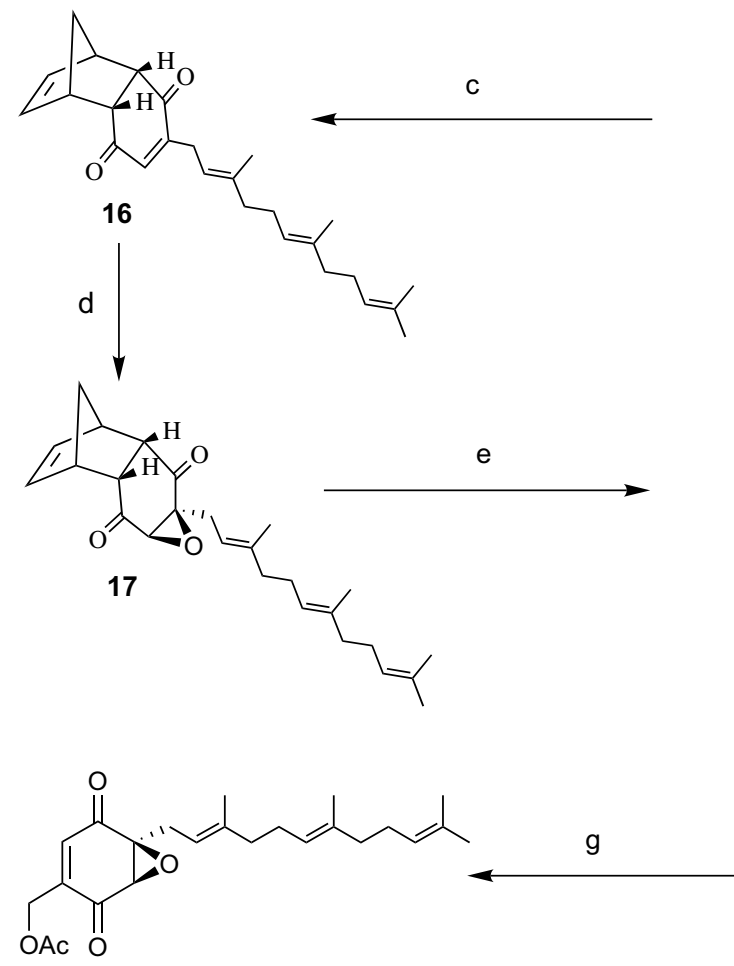

Yanuthone B 2<smiles>COc1ccc(O)c(C/C=C(\C)CC/C=C(\C)CCC=C(C)C)c1</smiles>

15

$\mathrm{b}$<smiles>CC(C)=CCC/C(C)=C/CC/C(C)=C/CC1=CC(=O)C=CC1=O</smiles>

12

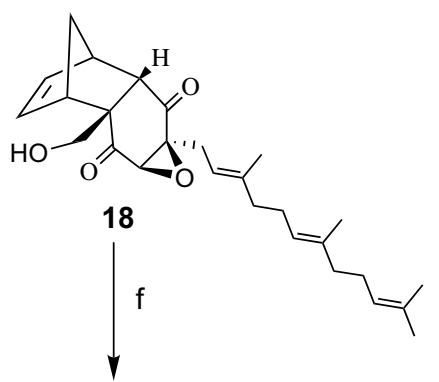<smiles></smiles>

19

Scheme 1. Reagents and conditions: (a) $(E, E)$-farnesol 14, $\mathrm{Sc}(\mathrm{OTf})_{3}(30 \mathrm{~mol} \%)$, toluene, $0-5{ }^{\circ} \mathrm{C}, 18 \mathrm{~h}, 45 \%$; (b) $\mathrm{CAN}_{2} \mathrm{CH}_{3} \mathrm{CN}-\mathrm{H}_{2} \mathrm{O}(1: 1), 0{ }^{\circ} \mathrm{C}$, $1.5 \mathrm{~h}, 76 \%$; (c) cyclopentadiene, $\mathrm{MeOH}, 0{ }^{\circ} \mathrm{C}, 2 \mathrm{~h}, 88 \%$; (d) $10 \% \mathrm{Na}_{2} \mathrm{CO}_{3}, 50 \% \mathrm{H}_{2} \mathrm{O}_{2}, 0{ }^{\circ} \mathrm{C}, 2 \mathrm{~h}, 85 \%$; (e) DBU, 35\% formalin, THF, $0{ }^{\circ} \mathrm{C}, 2 \mathrm{~h}, 86 \%$; (f) diphenyl ether, $240{ }^{\circ} \mathrm{C}, 6 \mathrm{~min}, 74 \%$; (g) $\mathrm{Ac}_{2} \mathrm{O}$, pyridine, DMAP, DCM, $0{ }^{\circ} \mathrm{C}, 90 \%$.

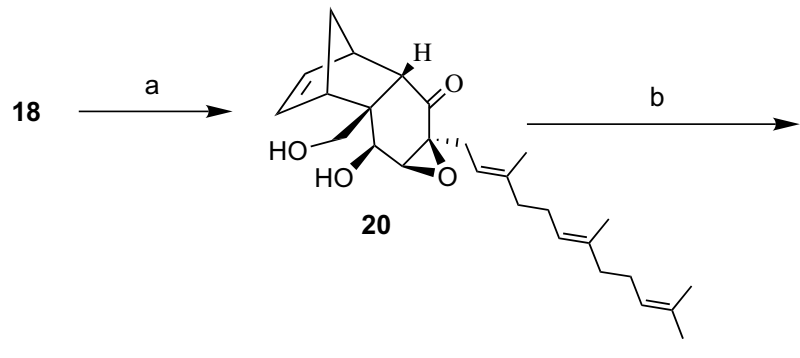

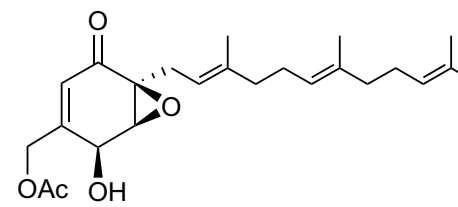

Yanuthone A 1<smiles>CC(C)=CCC/C(C)=C/CC/C(C)=C/CO[C@@]12O[C@H]1[C@H](O)C(CO)=CC2=O</smiles>

22- Deacetyl A 8 c<smiles>CC(=O)O[C@H]1C(CO)=CC(=O)[C@@H]2O[C@@H]12</smiles>

Yanuthone C 3

Scheme 2. Reagents and conditions: (a) DIBAL-H (2.0 equiv), THF, $-78{ }^{\circ} \mathrm{C}, 30 \mathrm{~min}, 62 \%$; (b) diphenylether, $240{ }^{\circ} \mathrm{C}, 12 \mathrm{~min}, 69^{\circ}$; (c) $\mathrm{Ac} \mathrm{c}_{2} \mathrm{O}$, pyridine, DCM, $-20{ }^{\circ} \mathrm{C}, 80 \%$ based on the recovery of S.M. 


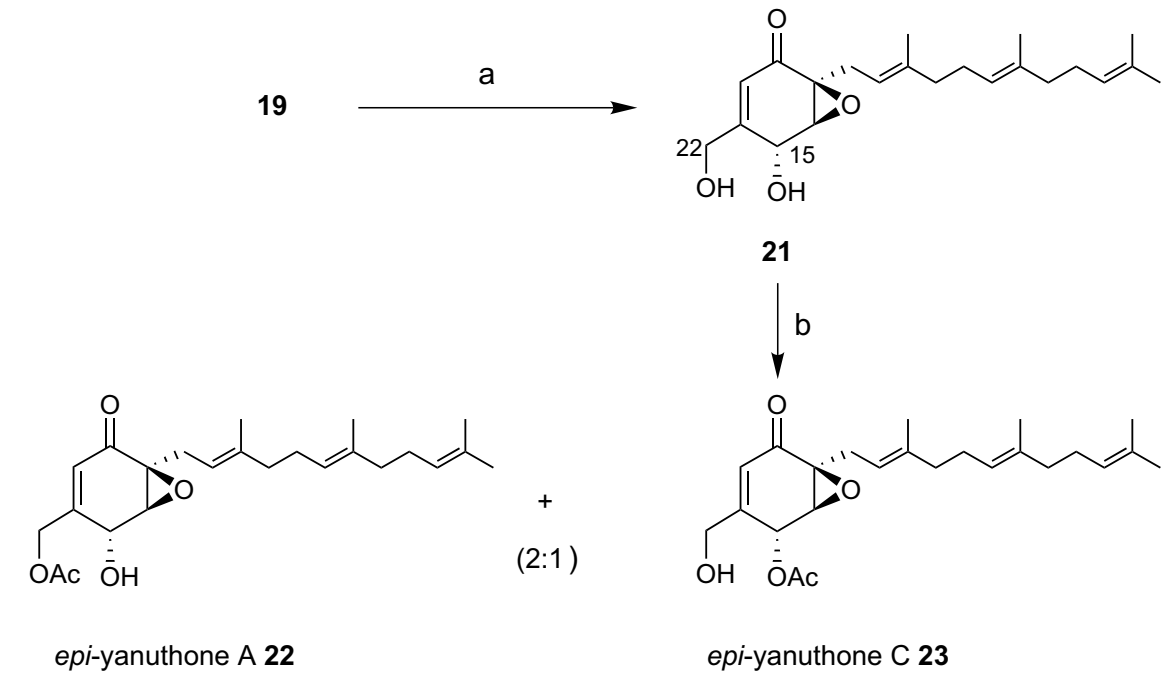

Scheme 3. Reagents and conditions: (a) DIBAL-H (2.0 equiv), THF, $-78{ }^{\circ} \mathrm{C}, 30 \mathrm{~min}, 71 \%$; (b) $\mathrm{Ac} \mathrm{C}_{2} \mathrm{O}$, pyridine, $\mathrm{DCM},-20{ }^{\circ} \mathrm{C}, 80 \%$ based on the recovery of S.M.

found to be in the range of $1.3-1.6 \mathrm{~Hz}$, indicating a trans relationship of these protons and fully secured the stereochemistry of the yanuthones.

In summary, we have accomplished the first total synthesis of the marine natural products yanuthones $\mathrm{A}-\mathrm{C}$ and 22-deacetylyanuthone A from commercially available $p$-methoxyphenol through the intermediacy of 2-farnesyl-p-benzoquinone involving a short, general and stereocontrolled sequence. The strategy outlined here is amenable to adaptation for the synthesis of other members of the farnesylated epoxycyclohexenoid family.

\section{Acknowledgements}

This work was supported by the Chemical Biology Unit of JNCASR at the Indian Institute of Science.

\section{References and notes}

1. (a) Mehta, G.; Singh, V. Chem. Soc. Rev. 2002, 31, 324 334; (b) Tietze, L. F.; Bell, H. P.; Chandrasekhar, S. Angew. Chem., Int. Ed. 2003, 42, 3996-4028.

2. Bugni, T. S.; Abbanat, D.; Bernan, V. S.; Maiese, W. M.; Greenstein, M.; Wagoner, R. M. V.; Ireland, C. M. J. Org. Chem. 2000, 65, 7195-7200.

3. Li, X.; Choi, H. D.; Kang, J. S.; Lee, C.; Son, B. W. J. Nat. Prod. 2003, 66, 1499-1500.

4. Stadler, M.; Sterner, O.; Anke, H. Z. Naturforsch. 1993, C48, 843-850.

5. Anderson, M. G.; Jarman, T. B.; Rickards, R. W. J. Antibiot. 1995, 48, 391-398.

6. Angela, P. L.; Eliseo, A.; Diaz, P. D.; Maria, A. Rev. Colom. Quim. 2000, 29, 25-37 (Chem. Abstr. 2000, 136, 229385).

7. (a) Bouzbouz, S.; Kirschleger, B.; Villieras, J. Bull. Soc. Chim. Fr. 1997, 134, 67-84; (b) Pearce, B. C. US Patent 5318993, 1994 (Chem. Abstr. 1994, 122, 16309).

8. (a) Ishibashi, H.; Ishihara, K.; Yamamoto, H. J. Am. Chem. Soc. 2004, 126, 11122-11123; (b) Tsuchimoto, T.; Tobita, K.; Hiyama, T.; Fukuzawa, S. J. Org. Chem. 1997, 62, 6997-7005.
9. All new compounds were characterized on the basis of IR, ${ }^{1} \mathrm{H}$ and ${ }^{13} \mathrm{C}$ NMR and HRMS data. Spectral data for selected compounds: compound 12: ${ }^{1} \mathrm{H}$ NMR $(300 \mathrm{MHz}$, $\left.\mathrm{CDCl}_{3}\right) \delta 6.78-6.68(\mathrm{~m}, 2 \mathrm{H}), 6.56-6.52(\mathrm{~m}, 1 \mathrm{H}), 5.18-5.07$ $(\mathrm{m}, 3 \mathrm{H}), 3.14(\mathrm{~d}, 2 \mathrm{H}, J=7.5 \mathrm{~Hz}), 2.14-1.97(\mathrm{~m}, 8 \mathrm{H}), 1.68(\mathrm{~s}$, $3 \mathrm{H}), 1.64(\mathrm{~s}, 3 \mathrm{H}), 1.60(\mathrm{~s}, 6 \mathrm{H}) ;{ }^{13} \mathrm{C} \mathrm{NMR}\left(75 \mathrm{MHz}, \mathrm{CDCl}_{3}\right)$ $\delta 187.9,187.6,148.5,140.2,136.7,136.3,135.5,132.3,131.3$, $124.3,123.7,117.6,39.6,27.4,26.7,26.4,25.7,17.7,16.2$ 16.1. Compound 18: ${ }^{1} \mathrm{H}$ NMR $\left(400 \mathrm{MHz}, \mathrm{CDCl}_{3}\right) \delta 6.07(\mathrm{~s}$, $1 \mathrm{H}), 6.05(\mathrm{~s}, 1 \mathrm{H}), 5.09-5.05(\mathrm{~m}, 2 \mathrm{H}), 4.92(\mathrm{t}, 1 \mathrm{H}, J=$ $5.4 \mathrm{~Hz}), 4.38(\mathrm{~d}, 1 \mathrm{H}, J=11.6 \mathrm{~Hz}), 3.81(\mathrm{~d}, 1 \mathrm{H}, J=11.2 \mathrm{~Hz})$, $3.42(\mathrm{~s}, 1 \mathrm{H}), 3.31(\mathrm{br} \mathrm{s}, 2 \mathrm{H}), 2.87(\mathrm{~d}, 1 \mathrm{H}, J=3.2 \mathrm{~Hz}), 2.64$ $2.62(\mathrm{~m}, 2 \mathrm{H}), 2.07-1.95(\mathrm{~m}, 8 \mathrm{H}), 1.69(\mathrm{~s}, 3 \mathrm{H}), 1.60(\mathrm{~s}, 9 \mathrm{H})$, $1.53(\mathrm{~d}, 1 \mathrm{H}, J=9.6 \mathrm{~Hz}), 1.46(\mathrm{~d}, 1 \mathrm{H}, J=9.6 \mathrm{~Hz}) ;{ }^{13} \mathrm{C} \mathrm{NMR}$ $\left(100 \mathrm{MHz}, \mathrm{CDCl}_{3}\right) \delta 205.8,204.7,140.9,138.1,138.0$, $135.4,131.5,124.3,123.7,114.8,68.2,67.8,61.8,53.9,45.9$, $44.3,43.4,39.7,26.7,26.4,25.8,25.6,17.7,16.4,16.1$; HRMS (ES) $m / z$ calcd for $\mathrm{C}_{27} \mathrm{H}_{36} \mathrm{O}_{4} \mathrm{Na}[\mathrm{M}+\mathrm{Na}]^{+}$: 447.2511, found: 447.2526. Compound 2: ${ }^{1} \mathrm{H}$ NMR $\left(300 \mathrm{MHz}, \mathrm{CDCl}_{3}\right) \delta 6.53(\mathrm{t}, 1 \mathrm{H}, J=1.8 \mathrm{~Hz}), 5.09-4.96$ $(\mathrm{m}, 3 \mathrm{H}), 4.96(\mathrm{dd}, 1 \mathrm{H}, J=1.8,16.8 \mathrm{~Hz}), 4.81(\mathrm{dd}, 1 \mathrm{H}$, $J=1.8,16.8 \mathrm{~Hz}), 3.67(\mathrm{~s}, 1 \mathrm{H}), 2.89(\mathrm{dd}, 1 \mathrm{H}, J=8.1$, $14.7 \mathrm{~Hz}), 2.63(\mathrm{dd}, 1 \mathrm{H}, J=6.9,14.7 \mathrm{~Hz}), 2.14(\mathrm{~s}, 3 \mathrm{H})$, 2.07-1.97 (m, 8H), $1.68(\mathrm{~s}, 3 \mathrm{H}), 1.64(\mathrm{~s}, 3 \mathrm{H}), 1.59(\mathrm{~s}, 6 \mathrm{H})$; ${ }^{13} \mathrm{C}$ NMR $\left(75 \mathrm{MHz}, \mathrm{CDCl}_{3}\right) \delta 192.1,192.0,169.8,143.1$, $141.2,135.5,132.3,131.3,124.3,123.7,114.7,62.4,59.4$, 57.5, 39.7, 26.7, 26.3, 25.7, 25.2, 20.6, 17.7, 16.4, 16.0; HRMS (ES) $m / z$ calcd for $\mathrm{C}_{24} \mathrm{H}_{32} \mathrm{O}_{5} \mathrm{Na}[\mathrm{M}+\mathrm{Na}]^{+}$: 423.2147, found: 423.2158. Compound 8: ${ }^{1} \mathrm{H}$ NMR $\left(500 \mathrm{MHz}, \mathrm{CDCl}_{3}\right) \delta 5.96(\mathrm{~s}, 1 \mathrm{H}), 5.14-5.05(\mathrm{~m}, 2 \mathrm{H}), 5.00$ $(\mathrm{t}, 1 \mathrm{H}, J=7.3 \mathrm{~Hz}), 4.68(\mathrm{~d}, 1 \mathrm{H}, J=8.4 \mathrm{~Hz}), 4.45(\mathrm{~d}, 1 \mathrm{H}$, $J=15.0 \mathrm{~Hz}), 4.39(\mathrm{~d}, 1 \mathrm{H}, J=15.0 \mathrm{~Hz}), 3.72(\mathrm{~d}, 1 \mathrm{H}$, $J=2.8 \mathrm{~Hz}), 2.81(\mathrm{dd}, 1 \mathrm{H}, J=8.0,15.5 \mathrm{~Hz}), 2.72(\mathrm{~d}$, $1 \mathrm{H}, J=8.5 \mathrm{~Hz}), 2.51(\mathrm{dd}, 1 \mathrm{H}, J=7.0,15.5 \mathrm{~Hz}), 2.27(\mathrm{br}$ $\mathrm{s}, 1 \mathrm{H}), 2.07-1.95(\mathrm{~m}, 8 \mathrm{H}), 1.68(\mathrm{~s}, 3 \mathrm{H}), 1.64(\mathrm{~s}, 3 \mathrm{H}), 1.60(\mathrm{~s}$, $3 \mathrm{H}), 1.59$ (s, 3H); ${ }^{13} \mathrm{C}$ NMR $\left(100 \mathrm{MHz}, \mathrm{CDCl}_{3}\right) \delta 193.7$, 156.7, 140.1, 135.3, 131.5, 124.3, 123.8, 120.9, 115.9, 66.0, 62.9, 61.4, 59.0, 39.8, 26.7, 26.4, 25.9, 25.8, 17.8, 16.4, 16.1; HRMS (ES) $\mathrm{m} / \mathrm{z}$ calcd for $\mathrm{C}_{22} \mathrm{H}_{32} \mathrm{O}_{4} \mathrm{Na}[\mathrm{M}+\mathrm{Na}]^{+}$: 383.2198, found: 383.2206. Compound 1: ${ }^{1} \mathrm{H}$ NMR $\left(500 \mathrm{MHz}, \mathrm{CDCl}_{3}\right) \delta 5.93(\mathrm{~d}, 1 \mathrm{H}, J=1.3 \mathrm{~Hz}), 5.11-5.06$ $(\mathrm{m}, 2 \mathrm{H}), 5.01(\mathrm{t}, 1 \mathrm{H}, J=7.4 \mathrm{~Hz}), 4.87(\mathrm{~d}, 1 \mathrm{H}, J=15.7 \mathrm{~Hz})$, $4.78(\mathrm{~d}, 1 \mathrm{H}, J=15.7 \mathrm{~Hz}), 4.61(\mathrm{~s}, 1 \mathrm{H}), 3.72(\mathrm{~d}, 1 \mathrm{H}$, $J=3.0 \mathrm{~Hz}), 2.80(\mathrm{dd}, 1 \mathrm{H}, J=8.0,15.5 \mathrm{~Hz}), 2.52(\mathrm{dd}, 1 \mathrm{H}$, $J=6.8,15.6 \mathrm{~Hz}), 2.13(\mathrm{~s}, 1 \mathrm{H}), 2.09-1.95(\mathrm{~m}, 8 \mathrm{H}), 1.68(\mathrm{~s}$, 
$3 \mathrm{H}), 1.64(\mathrm{~s}, 3 \mathrm{H}), 1.60(\mathrm{~s}, 3 \mathrm{H}), 1.59(\mathrm{~s}, 3 \mathrm{H}) ;{ }^{13} \mathrm{C} \mathrm{NMR}$ $\left(100 \mathrm{MHz}, \mathrm{CDCl}_{3}\right) \delta 193.2,170.5,151.8,140.2,135.3$, 131.5, 124.3, 123.8, 122.4, 115.7, 65.5, 62.9, 61.4, 58.9, 39.8, 26.7, 26.4, 25.7, 25.9, 20.8, 17.8, 16.4, 16.1; HRMS (ES) $\mathrm{m} / \mathrm{z}$ calcd for $\mathrm{C}_{24} \mathrm{H}_{34} \mathrm{O}_{5} \mathrm{Na}[\mathrm{M}+\mathrm{Na}]^{+}:$425.2304, found: 425.2288. Compound 3: ${ }^{1} \mathrm{H}$ NMR $\left(500 \mathrm{MHz}, \mathrm{CDCl}_{3}\right) \delta$ $6.14(\mathrm{~d}, 1 \mathrm{H}, J=1.6 \mathrm{~Hz}), 5.91(\mathrm{~d}, 1 \mathrm{H}, J=1.0 \mathrm{~Hz}), 5.35(\mathrm{~d}$, $1 \mathrm{H}, J=5.0 \mathrm{~Hz}), 5.13-5.07(\mathrm{~m}, 2 \mathrm{H}), 5.01(\mathrm{t}, 1 \mathrm{H}, J=6.8 \mathrm{~Hz})$, $4.27(\mathrm{~d}, 1 \mathrm{H}, J=16.0 \mathrm{~Hz}), 4.22(\mathrm{~d}, 1 \mathrm{H}, J=16.0 \mathrm{~Hz}), 3.71(\mathrm{~d}$, $1 \mathrm{H}, J=2.8 \mathrm{~Hz}), 2.83(\mathrm{dd}, 1 \mathrm{H}, J=8.0,15.5 \mathrm{~Hz}), 2.51(\mathrm{dd}$, $1 \mathrm{H}, J=7.2,15.5 \mathrm{~Hz}), 2.22(\mathrm{~s}, 3 \mathrm{H}), 2.13-1.97(\mathrm{~m}, 8 \mathrm{H}), 1.68$ $(\mathrm{s}, 3 \mathrm{H}), 1.64(\mathrm{~s}, 3 \mathrm{H}), 1.59(\mathrm{~s}, 3 \mathrm{H}), 1.57(\mathrm{~s}, 3 \mathrm{H}) ;{ }^{13} \mathrm{C} \mathrm{NMR}$ $\left(100 \mathrm{MHz}, \mathrm{CDCl}_{3}\right) \delta 193.4,170.2,152.4,140.0,134.9$, $129.7,124.7,124.0,123.5,122.4,115.4,66.8,61.6,60.2$, 55.7, 39.4, 26.9, 26.5, 25.5, 25.4, 20.5, 17.4, 16.1, 15.7; HRMS (ES) $m / z$ calcd for $\mathrm{C}_{24} \mathrm{H}_{34} \mathrm{O}_{5} \mathrm{Na}[\mathrm{M}+\mathrm{Na}]^{+}$: 425.2304, found: 425.2314. Compound 21: ${ }^{1} \mathrm{H}$ NMR $\left(500 \mathrm{MHz}, \mathrm{CDCl}_{3}\right) \delta 6.00(\mathrm{~s}, 1 \mathrm{H}), 5.09-5.03(\mathrm{~m}, 3 \mathrm{H}), 4.62$ (br s, 1H), $4.53(\mathrm{~d}, 1 \mathrm{H}, J=15.2 \mathrm{~Hz}), 4.34(\mathrm{~d}, 1 \mathrm{H}, J=$ $15.2 \mathrm{~Hz}), 3.67(\mathrm{~d}, 1 \mathrm{H}, J=1.4 \mathrm{~Hz}), 3.03(\mathrm{~d}, 1 \mathrm{H}, J=7.0 \mathrm{~Hz})$, $2.79(\mathrm{dd}, 1 \mathrm{H}, J=7.9,15.0 \mathrm{~Hz}), 2.56(\mathrm{dd}, 1 \mathrm{H}, J=6.6$, $15.0 \mathrm{~Hz}), 2.27$ (br s, 1H), 2.10-1.97 (m, 8H), $1.68(\mathrm{~s}, 3 \mathrm{H})$, $1.65(\mathrm{~s}, 3 \mathrm{H}), 1.60(\mathrm{~s}, 3 \mathrm{H}), 1.59(\mathrm{~s}, 3 \mathrm{H}) ;{ }^{13} \mathrm{C} \mathrm{NMR}(100 \mathrm{MHz}$, $\left.\mathrm{CDCl}_{3}\right) \delta 194.8,155.7,140.1,135.3,131.4,124.3,123.9$, $122.1,115.9,64.5,64.1,60.2,59.8,39.7,26.7,26.4,25.7$, 17.7, 16.3, 16.0. 22: ${ }^{1} \mathrm{H}$ NMR $\left(300 \mathrm{MHz}, \mathrm{CDCl}_{3}\right) \delta 6.01(\mathrm{~s}$, $1 \mathrm{H}), 5.09-5.02(\mathrm{~m}, 3 \mathrm{H}), 4.83(\mathrm{~d}, 1 \mathrm{H}, J=16.2 \mathrm{~Hz}), 4.77(\mathrm{~d}$, $1 \mathrm{H}, J=16.5 \mathrm{~Hz}), 4.55(\mathrm{~s}, 1 \mathrm{H}), 3.66(\mathrm{~s}, 1 \mathrm{H}), 2.81(\mathrm{dd}, 1 \mathrm{H}$, $J=8.4,15.6 \mathrm{~Hz}), 2.57$ (br s, $1 \mathrm{H}), 2.53(\mathrm{dd}, 1 \mathrm{H}, J=8.4$, $15.3 \mathrm{~Hz}), 2.14(\mathrm{~s}, 3 \mathrm{H}), 2.07-1.95(\mathrm{~m}, 8 \mathrm{H}), 1.68(\mathrm{~s}, 3 \mathrm{H}), 1.64$ $(\mathrm{s}, 3 \mathrm{H}), 1.60(\mathrm{~s}, 3 \mathrm{H}), 1.56(\mathrm{~s}, 3 \mathrm{H}) ;{ }^{13} \mathrm{C} \mathrm{NMR}(75 \mathrm{MHz}$, $\left.\mathrm{CDCl}_{3}\right) \delta 193.3,170.7,150.7,140.0,135.2,131.4,124.3$, $123.9,122.4,115.9,64.0,63.3,60.5,59.6,39.7,20.7,26.4$, 25.8, 25.7, 20.6, 17.7, 16.4, 16.1. Compound 23: ${ }^{1} \mathrm{H}$ NMR $\left(300 \mathrm{MHz}, \mathrm{CDCl}_{3}\right) \delta 6.19(\mathrm{~s}, 1 \mathrm{H}), 5.83(\mathrm{~s}, 1 \mathrm{H}), 5.18-4.98(\mathrm{~m}$, $3 \mathrm{H}), 4.25-4.18(\mathrm{~m}, 2 \mathrm{H}), 3.56(\mathrm{~d}, 1 \mathrm{H}, J=1.5 \mathrm{~Hz}), 2.80(\mathrm{dd}$, $1 \mathrm{H}, J=8.4,16.2 \mathrm{~Hz}), 2.56(\mathrm{dd}, 1 \mathrm{H}, J=6.9,15.6 \mathrm{~Hz}), 2.15$ $(\mathrm{s}, 3 \mathrm{H}), 2.10-1.95(\mathrm{~m}, 8 \mathrm{H}), 1.68(\mathrm{~s}, 3 \mathrm{H}), 1.63(\mathrm{~s}, 3 \mathrm{H}), 1.60(\mathrm{~s}$, $3 \mathrm{H}), 1.55(\mathrm{~s}, 3 \mathrm{H}) ;{ }^{13} \mathrm{C}$ NMR $\left(100 \mathrm{MHz}, \mathrm{CDCl}_{3}\right) \delta 193.4$, $170.3,151.5,140.0,135.2,131.4,124.3,124.2,123.8,115.9$, $64.2,62.5,59.5,58.2,39.7,26.7,26.5,25.7,25.5,20.7,17.7$, $16.3,16.0$.

10. (a) Mehta, G.; Islam, K. Tetrahedron Lett. 2003, 44, 3569-3572; (b) Mehta, G.; Ramesh, S. S. Tetrahedron
Lett. 2004, 45, 1985-1987; (c) Mehta, G.; Pan, S. C. Org. Lett. 2004, 6, 811-813; (d) Mehta, G.; Pan, S. C. Org. Lett. 2004, 6, 3985-3988; (e) Mehta, G.; Pan, S. C. Tetrahedron Lett. 2005, 46, 3045-3048.

11. Mehta, G.; Pujar, S. R.; Ramesh, S. S.; Islam, K. Tetrahedron Lett. 2005, 46, 3373-3376.

12. The stereo- and regiochemical outcome in the DIBAL-H reduction of 18 can be rationalized in terms of the initial coordination of aluminium with the hydroxyl group and the carbonyl group as shown below. This effectively blocks the preferred exo-face, precludes additional coordination of the epoxy oxygen with DIBAL-H and activates the carbonyl group for regioselective reduction. Consequently, reduction takes place from the hindered endo-face (see arrows) of the tricyclic system to deliver the observed product 20.

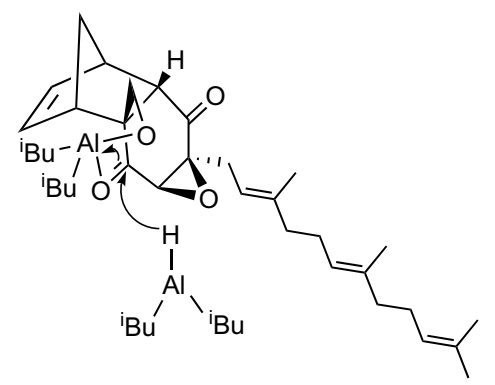

13. In the case of the more flexible epoxycyclohexenone 19, the regio- and stereochemical course of the DIBAL-H reduction is directed by coordination with the epoxy oxygen and intramolecular hydride delivery from the same face, as shown below, to result in the observed stereochemistry of $\mathbf{2 1}$.

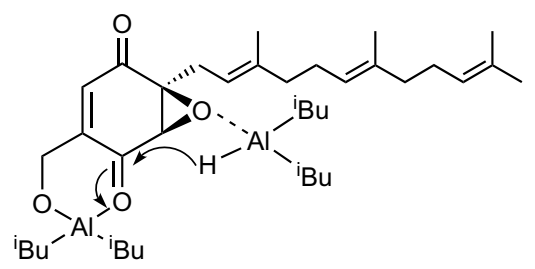

